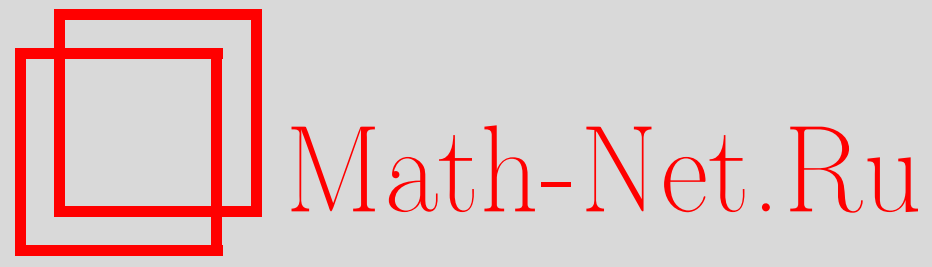

В. А. Зорич, Квазиконформные погружения римановых многообразий и теорема пикаровского типа, Функи. анализ и его прил., 2000, том 34, выпуск $3,37-48$

DOI: https://doi.org/10.4213/faa310

Использование Общероссийского математического портала Math$\mathrm{Net.Ru}$ подразумевает, что вы прочитали и согласны с пользовательским соглашением

http://www . mathnet.ru/rus/agreement

Параметры загрузки:

IP : 3.91 .87 .62

26 апреля 2023 г., 10:24:43

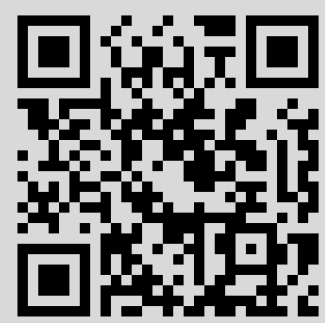


Функииональный анализ и его приложения

2000, т. 34, вып. 3, с. 37-48

УДК $517.54+514.774$

\title{
Квазиконформные погружения римановых многообразий и теорема пикаровского типа*
}

\author{
(c) 2000. В. А. Зорич
}

\section{$\S 1$. Формулировка основных результатов}

Цель этой работы - доказать теорему о продолжении ростка локально обратного отображения, которая могла бы служить инструментом исследования особенностей квазиконформных погружений римановых многообразий размерности $n \geqslant 3$.

Мы рассматриваем многообразия одной и той же размерности и, говоря о погружении, имеем в виду локально инъективное (локально обратимое) отображение. Квазиконформность погружения подразумевает ограниченность его коэффициента квазиконформности.

ТЕорема 1 (основная теорема). Пусть $M^{n}$ и $N^{n}$ - римановьл многообразия конечной размерности $n, \dot{U}=U \backslash\{\alpha\}$ - проколотая окрестность точки $\alpha \in M^{n}$

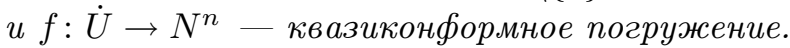

Если это отображение не инъективно ни в какой проколотой окрестности особой точки, то при $n \geqslant 3$ любой росток $f_{p}^{-1}$ локально обратного отображения продолжается вдоль любого пути на $N^{n}$, не задевающего образ $f(\partial U)$ точек края окрестности $U$.

Здесь под $f(\partial U)$ можно понимать предельное множество в $N^{n}$ отображения $f$ при подходе к $\partial U$; уменьшив окрестность, можно, однако, без ограничения общности считать, что $f$ определено на крае $\partial U$ и даже в его окрестности.

Доказательство теоремы 1 дано в $\S 4$.

Ниже (см. §6) мы приводим некоторые примеры ее приложений. Среди них - теорема о глобальном гомеоморфизме, а также геометрическая версия этой теоремы, предложенная М. Громовым.

В качестве попутного продукта получается также следующая

ТЕоремА 2 (пикаровского типа для квазиконформных погружений). Для квазиконформного погружения с изолированной особенностью одного риманова многообразия в другое риманово многообразие той же размерности $n \geqslant 3$ имеет место следуюшая альтернатива:

(а) либо отображение имеет предел (конечный или бесконечный) в изолированной особой точке,

(b) либо образ любой проколотой окрестности особой точки покрьвает все многообразие образа.

Отображение $z \mapsto \exp (1 / z)$ показывает, что условие $n \geqslant 3$ здесь существенно.

Вместе с тем обе возможности этой альтернативы реализуемы в любой размерности $n \geqslant 2$. Примером реализации может служить отображение Хопфа

*Работа выполнена при поддержке РФФИ, проект 99-01-01179. 
$f: \dot{\mathbb{R}}^{n}=\mathbb{R}^{n} \backslash\{o\} \rightarrow S^{n-1} \times S^{1}$, определяемое соответствием $\dot{\mathbb{R}}^{n} \ni x \mapsto\left\{2^{k} x:\right.$ $k \in \mathbb{Z}\} \in \dot{\mathbb{R}}^{n} / G$, где $G$ - группа гомотетий $g: \dot{\mathbb{R}}^{n} \rightarrow \dot{\mathbb{R}}^{n}$, порожденная отображением $x \mapsto 2 x$.

Доказательство основной теоремы опирается на ряд лемм. Ключевым является следующее вспомогательное утверждение о связи конформной емкости и геометрии видимой части границы звездной области.

ЛЕмма 1 (о звезде). Пусть $D \subset \mathbb{R}^{n}(n \geqslant 2)$ - область, звездная относительно точки о $\in \mathbb{R}^{n}$, т.е. D образована бесконечными лучами или их конечньии интервалами, исходящими из точки о и заканчивающимися (если интервал конечен) в точках (видимой из $\alpha$ ) части $\Delta$ граничь $\partial D$ области $D$.

Пусть $\Gamma$ - семейство всех кривых в $D$, соединяющих $\varepsilon$-окрестность $B(o, \varepsilon)$ точки о с $\Delta$.

Если конформный модуль семейства Г равен нулю, то

(i) чентральная проекиия множества $\Delta$ на сферу $\partial B(o, \varepsilon)$ не содержит отличных от точки связных компонент и

(ii) $\partial D$ локально не разбивает пространство, если $n>2$.

\section{$\S 2$. Некоторые определения и комментарий}

В этом параграфе (добавленном по совету рецензента) мы кратко напомним для удобства читателя некоторые используемые ниже понятия.

2.1. Квазиконформное отображение. Отображение $f: D \rightarrow \mathbb{R}^{n}$ области $D \subset \mathbb{R}^{n}$ называется $k$-квазиконформным в $D$, если величина

$$
k_{f}(x):=\limsup _{\delta \rightarrow 0+} \frac{\max \{|f(\xi)-f(x)|:|\xi-x|=\delta\}}{\min \{|f(\xi)-f(x)|:|\xi-x|=\delta\}}
$$

(называемая линейным отклонением или коэффиииентом квазиконформности отображения $f$ в точке $x \in D$ ) ограничена в $D$ и $k_{f}(x) \leqslant k$ почти во всех точках $x \in D$. Заметим, что $k=1$ тогда и только тогда, когда $f-$ конформное отображение. Отображение называют квазиконформным или отображением с ограниченным искажением, если оно $k$-квазиконформно при некотором $k$ $(1 \leqslant k<\infty)$. Определение квазиконформности остается в силе для отображений метрических пространств (с естественной заменой модулей на соответствующие расстояния) и, в частности, для отображений римановых многообразий, с которыми мы будем иметь дело.

Полезно заметить, что коэффициент квазиконформности, очевидно, инвариантен относительно конформной замены метрики.

2.2. Конформные инварианты. $\mathrm{ff} \Omega \mathrm{fffl} \Omega \varnothing \equiv$ Ufffffif. Конформной емкостью конденсатора $R\left(C_{0}, C_{1}\right)$ называется величина

$$
\operatorname{cap} R\left(C_{0}, C_{1}\right):=\inf \int_{M^{n}}|\nabla u|^{n} d v,
$$

где нижняя грань берется по всем неотрицательным функциям (потенциалам) $u: M^{n} \rightarrow \mathbb{R}_{+}$с обобщенными производными, таким, что $\left.u\right|_{C_{0}}=0,\left.u\right|_{C_{1}}=1$.

Например, конформная емкость конденсатора $R_{r_{0}}^{r_{1}} \subset \mathbb{R}^{n}$, ограниченного сферами $C_{0}=\left\{x \in \mathbb{R}^{n}:|x|=r_{0}\right\}, C_{1}=\left\{x \in \mathbb{R}^{n}:|x|=r_{1}\right\}$, где $0<r_{0}<r_{1}$, равна

$$
\operatorname{cap} R_{r_{0}}^{r_{1}}=\omega_{n-1} \ln ^{1-n}\left(r_{1} / r_{0}\right) \text {, }
$$

где $\omega_{n-1}$ - это $(n-1)$-мера (площадь) единичной сферы в $\mathbb{R}^{n}$. 
ff^ı ß. Конформная емкость конденсатора может быть описана несколько иначе, в терминах модуля (или экстремальной длины $[1,2])$ семейства кривых (или мер). Порой такая форма предпочтительна.

Пусть $\Gamma=\{\gamma\}$ - семейство кривых на $n$-мерном римановом многообразии $\left(M^{n}, g\right)$. Измеримая по Борелю неотрицательная функция $\rho: M^{n} \rightarrow \mathbb{R}_{+}$называется допустимой для $\Gamma$, если $\int_{\gamma} \rho d s \geqslant 1$ для каждой кривой $\gamma \in \Gamma$. Конформнылм модулем семейства кривых Г называется величина

$$
\bmod \Gamma:=\inf \int_{n} \rho^{n} d v
$$

где нижняя грань берется по всем допустимым для Г функциям.

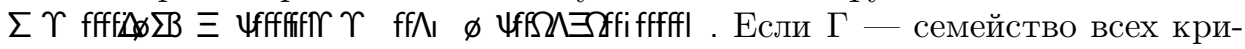
вых в $R\left(C_{0}, C_{1}\right)$, соединяющих граничные компоненты $C_{0}, C_{1}$ конденсатора $R\left(C_{0}, C_{1}\right)$, то

$$
\bmod (\Gamma)=\operatorname{cap} R\left(C_{0}, C_{1}\right)
$$

(См., например, $[3,4]$.$) Процесс осреднения (свертка с регулярным ядром) по-$ зволяет при вычислении емкости конденсатора и модуля соответствующего семейства кривых ограничиться функциями порядка гладкости самого многообразия, притом постоянными в окрестности компонент $C_{0}$ и $C_{1}$.

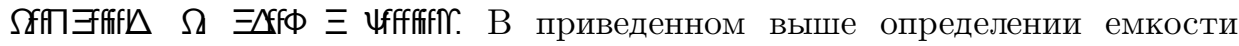
конденсатора его обкладки $C_{0}$ и $C_{1}$ могут быть достаточно общими множествами, не обязательно континуумами. Если множество $:=C_{1}$ таково, что $\operatorname{cap} R\left(C_{0}, \quad\right)=0$ для некоторого (а тогда и для любого) невырожденного и не пересекающего континуума $C_{0}$, то говорят, что множество имеет нулевую конформную емкость или что есть множество конформной емкости нуль.

Это равносильно тому, что модуль семейства всех кривых, пересекающих , равен нулю. Метрическая (хаусдорфова) размерность такого множества тоже равна нулю (см., например, [2]).

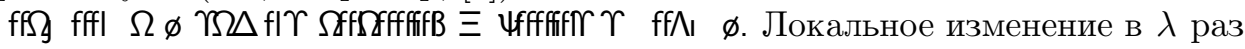
элемента длины $d \tilde{s}=\lambda(x) d s$ на римановом многообразии $\left(M^{n}, g\right)$, т. е. переход к конформно-эквивалентной метрике $\tilde{g}=\lambda^{2} g$ (что на физическом языке означает калибровочное преобразование, связанное с произволом в выборе местных масштабов), приводит к изменению в $\lambda^{n}$ раз численной величины элемента объема и в $\lambda^{-1}$ раз градиента функции; поэтому форма $|\nabla u|^{n} d v$, а вместе с ней и конформная емкость, инвариантны относительно конформного изменения метрики.

Аналогично, если в соответствии с изменением в $\lambda$ раз элемента длины на $\left(M^{n}, g\right)$ заменить $\rho$ новой допустимой функцией $\lambda^{-1} \rho$, то можно увидеть, что модуль семейства кривых — тоже конформный инвариант.

Нетрудно также проверить, что конформная емкость и конформный модуль квазиинвариантны (меняются не более чем в $k^{n-1}$ раз) при $k$-квазиконформном отображении.

Это, в частности, влечет за собой инвариантность по отношению к любым квазиконформным отображениям свойства объекта иметь емкость нуль или модуль нуль, что будет неоднократно использовано ниже. 
2.3. Конформный тип риманова многообразия. Некомпактные римановы (и даже субримановы) многообразия можно конформно-инвариантно разделить на два класса в соответствии с тем, какова конформная емкость абсолюта (бесконечности) этого многообразия (см. [5, 6]).

Некомпактное риманово многообразие $\left(M^{n}, g\right)$ будем называть многообразием конформно-параболического типа, если конформная емкость его идеальной границы (абсолюта, бесконечности) равна нулю (т.е. сар $R\left(C, \partial M_{\infty}^{n}\right)=0$ для отличного от точки континуума $\left.C \subset M^{n}\right)$; если же эта емкость положительна, то будем называть $\left(M^{n}, g\right)$ многообразием конформно-гиперболического типа.

Каноническими примерами являются пространства Евклида и Лобачевского соответственно (это легко проверить, используя приведенную выше формулу емкости сферического конденсатора и модель Пуанкаре пространства Лобачевского в шаре).

Такое разделение многообразий по указанным конформным типам, очевидно, инвариантно относительно конформных замен римановой метрики многообразия.

Конформный тип многообразия определяется лишь геометрией многообразия на бесконечности или, как говорят, асимптотической геометрией многообразия.

Конформная параболичность или гиперболичность многообразия (в соответствии с изложенным выше) на другом языке означает, что модуль семейства всех кривых на многообразии, выходящих на бесконечность (т. е. покидающих любую его компактную часть), равен нулю или положителен соответственно. Этим мы неоднократно воспользуемся.

\section{§3. Вспомогательные утверждения}

ПрЕДЛОЖЕНИЕ 1 (об асимптотических значениях и накрытии). Пусть $f: \dot{U} \rightarrow N^{n}$ - погружение (или даже открытое отображение) проколотой окрестности $\dot{U}$ точки о многообразия $M^{n}$ в связное многообразие $N^{n}$ той же конечной размерности $n$.

Если это погружение не имеет асимптотических значений в особой точке о, то для каждой проколотой окрестности $\dot{V} \subset \dot{U}$ точки о имеет место равенство $f(\dot{V})=N^{n}$.

Под асимптотическим значением подразумевается предел (конечный или бесконечный) отображения $f$ вдоль пути, идущего в особую точку. Конечный предел - это точка многообразия $N^{n}$. Предел считается бесконечным, если предельное множество отображения $f$ вдоль рассматриваемого пути пусто (для конечномерных многообразий это означает, что по мере того, как точка пути приближается на $M^{n}$ к особенности $o$, ее образ выходит на абсолют, т.е. окончательно покидает любой компакт в $\left.N^{n}\right)$.

Сформулированное предложение 1 очень общее и очень простое. Для его доказательства достаточно сделать следующее наблюдение относительно погружений вообще.

ЗАмечАнИЕ 1 . Пусть $f: M^{n} \rightarrow N^{n}-$ погружение. Если поднятие какогото пути, лежащего в $N^{n}$, встречает преграду, т. е. не продолжается за некоторую точку $p \in N^{n}$, то прообраз поднятой части пути не может иметь предела или непустого предельного множества в $M^{n}$. 
Иными словами, по мере того как точка самого пути в образе приближается к $p$, ее прообраз на накрывающем пути в $M^{n}$ обязан окончательно покидать любой компакт в $M^{n}$. В рассматриваемом здесь конечномерном случае это равносильно уходу на абсолют («на бесконечность»).

В развитие замечания 1 сделаем сразу еще одно общее наблюдение, относящееся к специфике погружений.

ЗАМЕЧАНИЕ 2. Пусть, как $u$ выле, $f: M^{n} \rightarrow N^{n}-$ погружение, и пусть в некоторой области $D \subset N^{n}$ нам удалось вьлделить однозначную ветвь $f_{D}^{-1}$ обратного отображения. Предположим, что отображение $f_{D}^{-1}$ имеет предел по каждой из двух последовательностей, сходящихся к одной и той же граничной точке р области D. Если одноименнье точки этих последовательностей можно соединить дугами, лежащими в $D$ и стягивающимися $к$ той же точке $p$ (m. е. попадающими полностью в сколь угодно малую окрестность точки $p$ ), то оба указанньх предела отображения $f_{D}^{-1}$ совпадают.

В частности, если область $D$ локально связна в граничной точке $p$ или если граница $\partial D$ области в окрестности точки $p$ вообще не разбивает пространство $\left(\right.$ как луч в $\mathbb{R}^{3}$, а не в $\left.\mathbb{R}^{2}\right)$, то это условие соединимости выполняется автоматически.

Добавим, наконец, что в этом последнем случае отображение $f_{D}^{-1}$ уже обязано иметь предел (конечный или бесконечный) в точке $p \in \partial D$.

Все утверждения, высказанные в замечаниях 1 и 2, основаны на том, что отображение $f$ локально инъективно и, в частности, не может отправлять в точку связное множество, отличное от точки.

Теперь для доказательства предложения 1 остается рассмотреть пересечение образов стягивающихся к $o \in M^{n}$ проколотых окрестностей этой точки. Если это пересечение не совпадает с $N^{n}$, то либо оно пусто, либо имеет граничную точку $p$ в $N^{n}$. Мы покажем, что ни то, ни другое невозможно.

Первое допущение противоречит отсутствию «бесконечных» асимптотических значений, и мы к этому еще вернемся. Рассмотрим второй случай. Учитывая, что $f$ открыто, уменьшив $\dot{U}$, можно считать, что $p \notin f(\partial U)$. Тогда в малой окрестности точки $p$ нет точек из $f(\partial U)$, но есть точки из $f(\dot{U})$. Поднятие идущего в $p$ пути, который не задевает $f(\partial U)$, не должно иметь препятствий, иначе возникнет конечное асимптотическое значение отображения $f$ в точке $о$. Следовательно, целая окрестность точки $p$ в $N^{n}$, не задевающая $f(\partial U)$, накрывается в том смысле, что ее прообраз в $\dot{U}^{n}$ состоит из гомеоморфных (посредством $f$ ) ей областей, накапливающихся к $\alpha$. Но тогда $p-$ не граничная точка рассмотренного пересечения и рассмотренный гипотетический случай невозможен.

Теперь вернемся к первому допущению и сделаем в этой связи следующее общее

ЗАмЕчАнИЕ 3. Если погружение $f: \dot{U} \rightarrow N^{n}$ не имеет конечных асимптотических значений в особой точке $\alpha \in M^{n}$, а пересечение образов стягивающихся к о проколотьх окрестностей этой точки не совпадает с $N^{n}$, то указанное пересечение пусто и $f$ имеет бесконечный предел в этой особой точке.

В самом деле, возьмем последовательность $\left\{\dot{u}_{m}\right\}$ проколотых $1 / m$-окрестностей особой точки $\alpha$. В образе мы получим последовательность вложенных 
связных множеств. Их пересечение, как мы только что показали, не имеет граничных точек в связном многообразии $N^{n}$. Нам дано, что оно не совпадает с $N^{n}$. Значит, оно пусто.

Наконец, если бы отображение $f$ не имело бесконечного предела в точке $\alpha$, то все образы $f\left(\dot{u}_{m}\right)$ имели бы непустое пересечение с некоторым компактом $K \subset N^{n}$. Рассмотрев последовательность вложенных компактов $K \cap \overline{f\left(\dot{u}_{m}\right)}$, мы нашли бы точку $p \in K$, граничную для $\bigcap f\left(\dot{u}_{m}\right)$. Но, как мы уже отметили, это невозможно, если погружение $f: \dot{U} \rightarrow N^{n}$ не имеет конечных асимптотических значений в точке $\alpha$.

Таким образом, замечание 3, а вместе с ним и предложение 1, доказаны.

Главным шагом в доказательстве основной теоремы, использующим квазиконформность погружения $f$ и условие $n \geqslant 3$ на размерность многообразий, является

ПРЕДЛОЖЕНИЕ 2 (о локальном кваЗиконформном продолжении). Пусть $M^{n}$, $N^{n}$ - связные римановь многообразия размерности $n, \dot{U}=U \backslash\{O\}-$ проколотая окрестность множества $O \subset M^{n}$ конформной емкости нуль (например, точки) и $f: \dot{U} \rightarrow N^{n}$ - квазиконформное погружение. Если $n \geqslant 3$, то для любой точки $a \in f(\dot{U}) \backslash f(\partial U)$ найдутся такая ее окрестность $W \subset N^{n}$ u такое множество $S$ конформной емкости нуль, что любой росток $f_{a}^{-1}$ локально обратного отображения в точке $a \in N^{n}$ продолжается вдоль любого пути в $W \backslash S$, а получаемая при этом ветвь обратного отображения однозначна в $W \backslash S$.

Сейчас мы обоснуем это утверждение, опираясь на сформулированную в конце $\S 1$ лемму (о звезде), которую докажем позже в $\S 5$.

Поскольку рассматривается вопрос лишь о локальном продолжении ростка (в окрестности точки $a \in N^{n}$ ), то без ограничения общности можно считать, что $N^{n}=\mathbb{R}^{n}$ и что точка $a$ находится в начале координат.

Пусть $W$ - шар с центром в $a$, такой, что $W \subset f(\dot{U}) \backslash f(\partial U)$. Продолжим росток $f_{a}^{-1}$ в пределах $W$ по лучам, исходящим из точки $a$, так далеко, как это только можно. Получим звездную область $D \subset W$, куда удалось продолжить росток $f_{a}^{-1}$ и получить в ней однозначную ветвь $f_{D}^{-1}$ обратного отображения. Часть $\Delta$ границы области $D$, находящаяся строго внутри $W$ и видимая из точки $a$, состоит из особых точек в том смысле, что через точки множества $\Delta$ продолжение оказалось невозможным. Это означает (см. доказательство предложения 1 ), что предельное множество отображения $f_{D}^{-1}$ в любой точке множества $\Delta$ пусто.

Поскольку $W \subset f(\dot{U}) \backslash f(\partial U)$, заключаем, что любой путь в области $D$, ведущий в одну из точек множества $\Delta$, под действием отображения $f_{D}^{-1}$ преобразуется в путь, который лежит в $\dot{U}$ и ведет именно в множество $O=U \backslash \dot{U}$ (а не в точки множества $\partial U$ ).

Конформная емкость множества $O$ по условию равна нулю $(\operatorname{cap}(O)=0)$. Значит, модуль всех кривых в $\mathbb{R}^{n}$, задевающих своим замыканием множество $O$, тоже равен нулю.

Отображение $f_{D}^{-1}$ квазиконформно. Значит, и модуль всех кривых в $D$, ведущих в точки из $\Delta$, равен нулю. 
По лемме о звезде (см. последний абзац 5 ) множество $\partial D \cap W$ локально не разделяет пространство. Значит, продолжая отображение $f_{D}^{-1}$ в точки множества $\partial D \cap W$ там, где это еще возможно, мы получим однозначное квазиконформное отображение $f_{\widetilde{D}}^{-1}$ в некоторой расширенной области $\widetilde{D} \subset W(\mathrm{~cm}$. замечание 2). По построению отображение $f_{\widetilde{D}}^{-1}$ уже не допускает продолжения в точки множества $S=\partial \widetilde{D} \cap W$. Значит, по замечанию 2 все эти точки особые, и, повторяя проведенное выше рассуждение, заключаем, что модуль семейства всех кривых в $\widetilde{D}$, замыкание которых задевает $S$, равен нулю. Тогда конформная емкость множества $S$ равна нулю. Различных ростков $f_{a}^{-1}$ может быть не более чем счетное число. Поскольку счетное объединение множеств $S$ емкости нуль является множеством емкости нуль, то предложение 2 доказано.

Известно, что если множество имеет конформную емкость нуль, то оно очень редкое, точнее, его метрическая размерность равна нулю. В определенном смысле $S$ является квазиконформным образом исходного особого множества $O$ (или его части); поэтому полученное свойство множества $S(\operatorname{cap}(S)=0)$ вполне естественно.

Отметим также, что если исходное множество $O$ состоит из единственной (изолированной особой) точки, то $S$ в пределах $W$ либо пусто, либо тоже точка.

Отображение $f_{\widetilde{D}}^{-1}$ квазиконформно продолжается в эту точку, а исходное отображение $f$ оказывается инъективным в проколотой окрестности точки $O$ и квазиконформно продолжаемым в эту точку .

Прямым следствием сказанного является такое

ПРЕДЛОЖЕНИЕ 3 (об асимптотических значениях и устранимости особенности квазиконформного погружения). Пусть $M^{n}, N^{n}-$ римановь многообра-

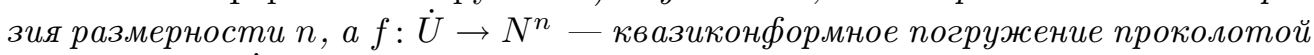
окрестности $\dot{U}=U \backslash\{о\}$ точки о $\in M^{n}$.

Если точка о не является устранимой особенностью погружения $f$, то при $n \geqslant 3$ отображение не имеет конечных асимптотических значений в этой особой точке.

Напомним, что выше в определении асимптотического значения мы допускали как конечный, так и бесконечный пределы.

\section{$\S 4$. Доказательство и обобщение основной теоремы}

Доказывая предложение 1 , мы показали, что если поднятие в $\dot{U} \subset M^{n}$ некоторого пути $\gamma \subset N^{n}$ встречает препятствие $p \in N^{n}$, то поднятая часть пути $\gamma$ обязана идти либо в особую точку $\alpha \in U$, либо к краю $\partial U$ окрестности $U$.

Но если $\gamma \cap f(\partial U)=\varnothing$, то остается только первая возможность. Значит, $p$ оказывается асимптотическим значением отображения $f$ в точке $о$. В силу предложения 3 отображение $f$ обязано тогда быть инъективным в некоторой проколотой окрестности точки о. Теорема 1 доказана.

Как видно, существенным в доказательстве теоремы 1, наряду с условием $n \geqslant 3$, было только то, что выходящее на особенность $о$ семейство кривых имело равный нулю конформный модуль и что образ этого семейства при погружении $f$ тоже имел конформный модуль, равный нулю.

Эти обстоятельства, с одной стороны, допускают расширение самого класса погружений $f$, например, можно считать $f$ квазиконформным в среднем, а с 
другой стороны, позволяют сформулировать основную теорему в следующем более общем виде

ТЕОРемА 3 (о квазиконформном погружении многообразий конформно-параболического типа). Пусть $U^{n}$ есть $n$-мерное риманово многообразие с компактным (возможно, пустьмм) краем $\partial U^{n}$. Пусть $U^{n}$ имеет конформно-параболический mun, и пусть $f: U^{n} \rightarrow N^{n}-$ квазиконформное погружение многообразия $U^{n}$ в $n$-мерное риманово многообразие $N^{n}$.

Если $n \geqslant 3$, то любой росток $f_{p}^{-1}$ локально обратного отображения продолжается вдоль любого пути на $N^{n}$, не задевающего образа $f\left(\partial U^{n}\right)$ края u, быть может, еще некоторого множества $S$ особенностей, имеющего конбормную емкость ( и метрическую размерность) нуль.

В самом деле, конформная параболичность многообразия означает (см. 2 или [5]), что его конформная емкость в бесконечности равна нулю. Это равносильно тому, что конформный модуль семейства всех кривых, лежащих в $U^{n}$ и выходящих на бесконечность (т. е. покидающих любой компакт в $U^{n}$ ), равен нулю.

Этого достаточно для применения леммы о звезде и получения описанным в доказательстве основной теоремы методом однозначной ветви обратного отображения, определенной в односвязной области $V$ всюду, за исключением некоторого множества $S_{V}$, приближение к точкам которого уводит накрывающий путь в прообразе на абсолют. Ввиду параболичности многообразия $U^{n}$ и квазиинвариантности конформного модуля при квазиконформном отображении заключаем, что множество $S_{V} \subset V$ имеет конформную емкость нуль. Из общей теории известно (см., например, [2]), что тогда и метрическая (в смысле Хаусдорфа) размерность множества $S_{V}$ тоже равна нулю.

В размерности $n \geqslant 3$ удаление такого множества из области $V$, очевидно, не влияет на фундаментальную группу и не нарушает односвязности области $V$, если $V$ исходно была таковой. При $n=2$ удаление уже одной точки приводит к появлению нетривиальных циклов и многозначных ветвей при продолжении ростков отображений вдоль этих циклов.

Объединение счетного числа множеств емкости нуль также имеет емкость нуль. Взяв на $N^{n}$, точнее, на $N^{n} \backslash f(\partial U)$ счетную базу окрестностей типа $V$ и объединяя отвечающие им (и счетному числу возможных прообразов) особые множества $S_{V}$, получим множество $S$ емкости нуль. По построению любой путь в $N^{n}$, не задевающий $f(\partial U)$ и $S$, с началом в точке $p \in f(V) \subset N^{n}$ накрывается путем с началом в любой из точек $f^{-1}(p) \in U^{n}$.

\section{§5. Доказательство леммы о звезде}

Напомним сначала одно утверждение о емкости из работы [7], которым мы воспользуемся ниже. В [7] оно сформулировано и доказано для случая размерности $n=3$, но и формулировка, и доказательство переносятся на любую конечную размерность $n \geqslant 2$.

ЛЕмма 2 (об оценке конформного модуля). Пусть $\bigcup_{x \in I} D(x, r(x))$ - объединение $(n-1)$-мерных дисков $D(x, r(x))(x$ - иентр, $r(x)$ - радиус), расположенных в параллельных гиперплоскостях, ортогональньх отрезку I в пространстве $\mathbb{R}^{n}(n \geqslant 2)$. 
На $(n-2)$-мерном крае каждого диска $D(x, r(x))$ конечного радиуса фиксируется точка $x^{*}=x^{*}(x)$ и рассматривается семейство $\Gamma(x)$ всех кривых в $D(x, r(x))$ с концами $x$ u $x^{*}(x)$. Пусть $\Gamma=\bigcup_{x \in I} \Gamma(x)$.

Тогда

$$
\bmod \Gamma \geqslant c(n) \frac{\operatorname{mes} E_{r}}{r},
$$

где $E_{r}:=\{x \in I: r(x)<r\}$, mes $E_{r}$ - длина (внутренняя 1 -мера) множества $r \subset I$, a $c(n)$ - положительная постоянная, зависящая только от $n$.

Чтобы для удобства читателя сделать статью замкнутой, по совету рецензента укажем идею доказательства этой леммы.

Нам надо оценить снизу интеграл $\int \rho^{n} d v$ по области $\bigcup_{x \in I} D(x, r(x))$, где $\rho$ - функция, допустимая для Г. По теореме Фубини представим этот интеграл в виде повторного интеграла, взятого сначала по $D(x, r(x))$, а затем по $I$.

Оценим снизу интеграл по $(n-1)$-мерному диску $D:=D(x, r(x))$. Для упрощения обозначений будем считать, что $x=\alpha$, a $r(x)=r_{0}$.

Вводя в $D$ полярные координаты и применяя неравенство Гёльдера, имеем

$$
\int_{D} \rho^{n} d v=\int_{\Omega} d \omega \int_{0}^{r_{0}} \rho^{n} r^{n-2} d r \geqslant \int_{\Omega} d \omega\left(\int_{0}^{r_{0}} \rho d r\right)^{n}\left(\int_{0}^{r_{0}} r^{-(n-2) /(n-1)} d r\right)^{-(n-1)} .
$$

Если бы функция $\rho$ была допустимой для любого радиуса диска $D$, то мы имели бы $\int_{0}^{r_{0}} \rho d r \geqslant 1$ и немедленно получили бы нужную нам оценку, явно интегрируя и оценивая оставшуюся часть интеграла.

Нам же дано только, что $\rho$ допустима для кривых, идущих из центра $о$ в выделенную точку $x^{*}$ на граничной сфере диска $D$. Рассмотрим тогда подобласть $G$ в $D$, составленную из двух конусов с вершинами $o$ и $x^{*}$ соответственно и с общим основанием - сечением диска $D$ гиперплоскостью, перпендикулярной радиусу $\left[o, x^{*}\right]$ и проходящей через его середину. Область $G$ расслаивается на симметричные относительно указанной гиперплоскости двузвенные ломаные с концами $о$ и $x^{*}$. Интеграл от $\rho$ по каждой такой ломаной уже не меньше 1 . Этого достаточно, чтобы интеграл по области $G$, а тогда, тем более, и по диску $D$ имел нужную оценку снизу. Так доказывается лемма 2.

Теперь докажем лемму о звезде, сформулированную в $\S 1$.

Допустим, что центральная проекция множества особенностей $\Delta$ имеет отличную от точки связную компоненту $\delta$ на сфере $S=\partial B(o, \varepsilon)$. Тогда можно выбрать отрезок $I \subset \mathbb{R}^{n}$ с центром в $\alpha \in \mathbb{R}^{n}$ так, что ортогональная проекция множества $\delta$ на направление отрезка $I$ покрывает весь этот отрезок. Рассмотрим $(n-1)$-мерные конусы с вершиной $\alpha$ и осью, направленной вдоль $I$. Каждая такая коническая гиперповерхность вполне определяется углом между образующей конуса и фиксированным направлением отрезка $I$. По построению найдется такой интервал углов $[\alpha, \beta], 0<\alpha<\beta<\pi / 2$, что каждая коническая поверхность, отвечающая углу из этого интервала, пересекает множество $\delta \subset S$. Рассмотрим область $G \subset \mathbb{R}^{n}$, лежащую вне шара $B(o, \varepsilon)$ и ограниченную коническими поверхностями, соответствующими углам $\alpha$ и $\beta$, и высекаемой ими частью $S_{\alpha}^{\beta}$ сферы $S$. Область $G$ допускает явно выписываемое осесимметричное квазиконформное отображение, при котором конические поверхности ложатся в параллельные гиперплоскости, а $S_{\alpha}^{\beta}$ переходит в цилиндрическую поверхность $Z$ с образующей, параллельной направлению отрезка $I$. 
Модуль семейства кривых является квазиинвариантом квазиконформных отображений. Значит, если про исходное семейство Г (кривых, соединяющих $S$ и $\Delta)$ известно, что $\bmod \Gamma=0$, то это верно и для попавшего в $G$ подсемейства $\Gamma_{G} \subset \Gamma$, а также для его образа $\widetilde{\Gamma}_{G}$ при указанном выпрямляющем конусы отображении. Покажем, однако, что при сделанном нами допущении о том, что множество $\delta$ - не точечная компонента проекции множества $\Delta$ на $S$, получается, что $\bmod \widetilde{\Gamma}_{G}>0$.

Действительно, возьмем в $\widetilde{\Gamma}_{G}$ только те кривые, каждая из которых лежит в одной из параллельных гиперплоскостей, полученных из конических поверхностей.

Семейство $\bar{\Gamma}$ этих кривых составляет часть семейства $\widetilde{\Gamma}_{G} ;$ поэтому $\bmod \bar{\Gamma} \leqslant$ $\bmod \widetilde{\Gamma}_{G}$.

Однако семейство $\bar{\Gamma}$ можно считать полученным сокращением кривых того семейства $Г$, которое фигурирует в цитированной выше лемме об оценке конформного модуля. Значит, $\bmod \bar{\Gamma} \geqslant \bmod \Gamma$. Но, с учетом указанной в этой лемме оценки величины $\bmod \Gamma$, проделанное построение при допущении о невырожденности $\delta$ приводит к тому, что $\bmod \bar{\Gamma}>0$. Это противоречие завершает доказательство леммы о звезде.

Как видно из приведенного доказательства, оба утверждения леммы 1 остаются в силе, если вместо всего пространства $\mathbb{R}^{n}$ взять шар $B^{n}$ конечного радиуса с центром $\alpha \in \mathbb{R}^{n}$ и считать, что звездная относительно центра $\alpha$ область $D$ лежит в шаре $B^{n}$ и образована радиусами этого шара или их собственными отрезками, концы которых, отличные от $\alpha$, порождают множество $\Delta \subset B^{n}$.

\section{§6. Некоторые следствия}

6.1. Теорема о глобальном гомеоморфизме. Теорема о глобальном гомеоморфизме для квазиконформных отображений отражает следующее специфически многомерное явление (замечено М. А. Лаврентьевым, сформулировано в [8] для $n=3$; доказано позднее в [7]):

Если локально гомеоморфное отображение $f: \mathbb{R}^{n} \rightarrow \mathbb{R}^{n}$ квазиконформно, то при $n \geqslant 3$ оно гомеоморфно и $f\left(\mathbb{R}^{n}\right)=\mathbb{R}^{n}$.

Сформулированное утверждение можно теперь получить как следствие доказанной выше основной теоремы.

Действительно, с точностью до стереографической проекции можно, например, считать, что мы имеем погружение $\tilde{f}: \dot{S}^{n} \rightarrow \dot{S}^{n} \subset S^{n}$ проколотой $n$-мерной сферы в себя или в полную сферу $S^{n}$. Поскольку $n \geqslant 3$, на основании доказанной теоремы росток обратного отображения допускает продолжение в $S^{n}$ по любому пути, не задевающему, быть может, только одной точки.

Значит, обратное отображение однозначно определено вне этой точки. Но тогда отображение $\tilde{f}$ инъективно и $\tilde{f}\left(\dot{S}^{n}\right)$ гомеоморфно проколотой сфере. Возвращаясь к отображению $f$ и учитывая, что $f\left(\mathbb{R}^{n}\right) \subset \mathbb{R}^{n}$, заключаем, что $f-$ гомеоморфизм и $f\left(\mathbb{R}^{n}\right)=\mathbb{R}^{n}$.

(Отметим, что вопрос о глобальной обратимости локально обратимых полиномиальных отображений пространства $\mathbb{C}^{n}$ в себя, так называемая проблема якобиана, остается открытым. Голоморфность и квазиконформность в высших размерностях - почти ортогональные свойства.) 


\section{2. Геометрическая версия Громова теоремы о глобальном гомео-} морфизме. Теорема о глобальном гомеоморфизме имеет ряд нетривиальных обобщений уже в рамках $\mathbb{R}^{n}$ (см. обзор [9]).

С. П. Новиков поставил следующий общий вопрос. Рассмотрим квазиконформное погружение (иммерсию) $f: M^{n} \rightarrow N^{n}$ одного риманова многообразия в другое риманово многообразие той же размерности $n \geqslant 3$. Для каких $M^{n}$ и $N^{n}$ справедлива теорема о глобальном гомеоморфизме?

М. Громов $[10,11]$ в следующим виде распространил теорему о глобальном гомеоморфизме (свойство инъективности) на квазиконформные погружения римановых многообразий и частично ответил на этот вопрос:

Если $f: M^{n} \rightarrow N^{n}-$ квазиконформное погружение полного риманова многообразия $M^{n}$ конечного объема в односвязное риманово многообразие $N^{n}$ той же размерности $n$, то при $n \geqslant 3$ это отображение инъективно и хаусдорфова размерность множества $N^{n} \backslash f\left(M^{n}\right)$ равна нулю.

Доказательство, по-видимому, так и не было опубликовано; поэтому приводимый ниже его вариант, наверное, является первым записанным текстом на этот счет.

Для доказательства этого утверждения можно воспользоваться обобщением основной теоремы, данным выше в $\S 4$. Дело в том, что условие конечности объема для полного многообразия $M^{n}$, как легко проверить, означает, что многообразие $M^{n}$ имеет конформно-параболический тип. Тогда по теореме 3 росток обратного отображения продолжается вдоль любого пути на $N^{n}$, не задевающего некоторого (возможно пустого) множества конформной емкости нуль.

По условию $N^{n}$ односвязно, а так как $n \geqslant 3$, то удаление такого множества из $N^{n}$ не нарушает односвязности. Значит, построенная ветвь обратного отображения однозначна. Но тогда исходное отображение $f$ инъективно. По построению множество $N^{n} \backslash f\left(M^{n}\right)$ имеет конформную емкость нуль. Из общей теории известно (см., например, [2]), что тогда его метрическая размерность равна нулю.

6.3. Теорема Пикара для квазиконформных погружений. Докажем теперь сформулированную в $\oint 1$ теорему 2 (альтернативу) пикаровского типа о поведении квазиконформного погружения около изолированной особой точки в случае, когда размерность рассматриваемых многообразий больше двух.

В соответствии с предложением 3 достаточно рассмотреть случай, когда отображение $f$ не имеет конечных асимптотических значений в особой точке $o$.

Но тогда в силу замечания 3 либо отображение имеет бесконечный предел в точке $о$, либо образ любой проколотой окрестности этой точки совпадает со всем многообразием $N^{n}$, куда идет отображение. Этим доказательство теоремы 2 завершено.

\section{$\S 7$. Перспективы}

Дальнейший анализ основной теоремы 1, использующий развитую здесь технику и полученные результаты, показывает, что реализация конкретно одной из двух возможностей поведения квазиконформного погружения в изолированной особой точке существенно связана с глобальной топологией пространства $N^{n}$, куда идет отображение, которая пока оставалась в тени. А именно, при $n>2$ ситуация такова: если многообразие $N^{n}$ односвязно, то в некоторой проколотой окрестности особой точки непременно реализуется инъективность 
отображения. Более того, указанная инъективность может отсутствовать только тогда, когда многообразие $N^{n}$ содержится в серии многообразий очень специального топологического типа.

Мы предполагаем рассмотреть эти вопросы в другой работе.

Благодарности. Я признателен Математическому институту им. Макса Планка (MPIM) в Германии и Институту высших научных исследований (IHES) во Франции за гостеприимство во время подготовки этой работы, а также Российскому фонду фундаментальных исследований (РФФИ) за финансовую поддержку.

\section{ЛИТЕРАТУРА}

1. Ahlfors L., Beurling A. Conformal invariants and function-theoretic null-sets. Acta Math., 83, 101-129 (1950).

2. Fuglede B. Extremal length and functional completion. Acta Math., 98, 171-219 (1957).

3. Gehring F. W. Extremal length definitions for the conformal capacity of rings in space. Michigan Math. J., 9, 137-150 (1962).

4. Aikawa H., Ohtsuka M. Extremal length of vector measures. Ann. Acad. Sci. Fenn. Math., 24, No. 1, 61-89 (1999).

5. Зорич B. A., Кесельман B. М. О конформном типе риманова многообразия. Функц. анализ и его прил., 30, вып. 2, 40-55 (1996).

6. Zorich V. A. Asymptotic geometry and conformal types of Carnot-Carathéodory spaces. Geom. Funct. Anal., 9, No. 2, 393-411 (1999).

7. Зорич B. A. Теорема М. А. Лаврентьева о квазиконформных отображениях пространства. Матем. сб., 74, 417-433 (1967).

8. Лаврентьев M. А. Об одном дифференциальном признаке гомеоморфных отображений трехмерных областей. ДАН СССР, 20, 241-242 (1938).

9. Zorich $V$. A. The global homeomorphism theorem for space quasiconformal mappings, its development and related open problems. In: Lecture Notes in Math., vol. 1508, Springer-Verlag, Berlin, 1992, pp. 131-148.

10. Gromov M. Hyperbolic manifolds, groups and actions. Proceedings of the 1978 Stony Brook Conference, Ann. Math. Stud., vol. 97, Princeton Univ. Press; Princeton, N.J., 1981, pp. 183-213.

11. Gromov M. with appendices by Katz M., Pansu P., and Semmes S. Metric structures for Riemannian and non-Riemannian spaces. Birkhäuser, Boston-Basel-Berlin, 1999.

Московский государственный университет, механико-математический факультет

Поступило в редакцию e-mail vzor@online.ru

18 декабря 1998 г. 